\title{
The Effect of Sensory Stimulation versus Motor Control Cueing of the Foot on Balance in Elderly Females: Single Group Pretest-Posttest Study
}

\author{
Sarah Wilmot, Amanda Alfonso, Jessica Velez, Ayse 0zcan Edeer* \\ Dominican College Doctor of Physical Therapy Program, Orangeburg, NY, USA \\ Email: *ayse.edeer@dc.edu
}

How to cite this paper: Wilmot, S., Alfonso, A., Velez, J. and Edeer, A.O. (2019) The Effect of Sensory Stimulation versus Motor Control Cueing of the Foot on Balance in Elderly Females: Single Group Pretest-Posttest Study. Advances in Aging Research, 8, 119-128.

https://doi.org/10.4236/aar.2019.86009

Received: September 30, 2019

Accepted: October 28, 2019

Published: October 31, 2019

Copyright $\odot 2019$ by author(s) and Scientific Research Publishing Inc. This work is licensed under the Creative Commons Attribution International License (CC BY 4.0).

http://creativecommons.org/licenses/by/4.0/

(c) (i) Open Access

\begin{abstract}
Study Design: A Quasi Experimental Single Group Pre-Test Post-Test. Objectives: The aim of this study was to determine the effects of sensory stimulation and motor control cueing of the foot on balance in females over the age of 65. Background: Balance deficits in the elderly population have shown to have detrimental consequences. Evidence suggests that sensory stimulation and motor control of the foot improve postural sway, however, studies have yet to compare the effects of both interventions on balance. Method and Measures: A total of 40 participants were contacted to complete the study with 32 participants meeting inclusion criteria for data collection and completing the research study. Two data collection days were utilized to perform pre and post-test outcome measures using the Functional Reach Test and Overall Sway Index (collected using the Biodex Medical System Inc., Biodex Biosway Portable Balance System). The interventions were 2-minute sensory stimulation to the sole of each foot on day one, and the "short foot" motor control cue on day two. Four related sample T-tests were conducted with the interventions as the dependent variable and the time of observation (pre and post) as the independent variable. Results: The results revealed sensory stimulation significantly improved balance as indicated by a decline in postural sway from pretest $(5.92 \pm 3.05)$ to posttest $(5.00 \pm 2.92)(\mathrm{p}=0.045)$ and a significant increase in Functional Reach from pretest $(8.01 \pm 2.55)$ to posttest $(8.70 \pm 2.66)(\mathrm{p}<0.001)$. Motor control cueing demonstrated mixed results on balance with an increase in postural sway from pretest $(5.52 \pm 3.50)$ to posttest $(6.66 \pm 4.06)(\mathrm{p}=0.015)$, and an increase in Functional Reach from pretest $(8.47 \pm 2.34)$ to posttest $(9.01 \pm 2.37)(p=0.004)$. Conclusion: Based on the results, researchers concluded that sensory stimulation is a viable intervention to utilize in order to improve balance in the elderly population. Further research to identify the appropriate prescription of sensory stimulation to maximize effects should be completed.
\end{abstract}




\section{Keywords}

Older Adults, Female, Sensory, Balance

\section{Background}

The focus of our research is to determine which intervention has a greater measurable improvement on balance of the elderly female population, sensory stimulation or motor control cueing of the foot. Delving deeper into this research topic we can help to determine if neurological losses due to the natural aging process can be restored with physical therapy intervention and therefore help elderly patients regain age-related balance losses.

Balance is one of the most common concerns for the elderly population as age-related balance losses put this population at a significantly higher risk of falling [1]. For those over the age of 65 years old, falls are the leading cause of injury. Approximately 1 in 3 elderly people fall each year; of those, about $25 \%$ sustain a serious injury [2]. Falls in this population have devastating consequences, therefore the importance of developing techniques that help to increase balance in this population and decrease falls is crucial.

The use of afferent stimulation for the effect it has on proprioception and balance has been researched in the elderly population. With losses to the somatosensory system, there is an increased risk of falling or losing one's balance [3]. Researchers Priplata et al. [3] provided somatosensory feedback in the form of vibrating insoles to identify the effect it has on postural sway during static balance in elderly people. Priplata et al. [3] compared elderly to young participants, to see the impact of sensory feedback on postural sway. The researchers identified that the effect of the vibrating insoles was a decrease in postural sway in both participant populations, but greater in the elderly population and may lead to improved balance.

As supported by Priplata et al. [3], a study conducted by Mildren and Bent [4] also used vibration insoles to assess the effect sensory stimulation has on mechanoreceptors of the foot in relation to balance. This study used vibrations of two different frequencies and performed a joint-matching task to see the effect this vibration has on position sense. The two frequencies were applied to specific regions of the foot including two planter locations, the heel and distal metatarsals, and the proximal aspect of the dorsum of the foot. The study found greater effects on proprioception when vibration was applied at the heel, despite the frequency. The most influence the vibrations had on position sense was when the foot was passively plantarflexed. The results of sensory stimulation on proprioception in dorsiflexion were not clinically significant.

Rothermel et al. [5] designed a study to compare the outcome of a traditional balance program to a balance program that stressed the importance of foot positioning in improving postural control. The results of the research noted that 
postural control improved in the traditional balance program group and not the program emphasizing foot positioning [5].

Jung et al. [6] referenced Rothermel et al. [5] for the position of the subject when performing exercises to elevate the medial longitudinal arch. Foot positioning must be cued to perform, therefore, utilizing the findings of this study to properly activate the intended intrinsic stabilizer is pertinent to our research.

The purpose of our research is to determine whether sensory stimulation or motor control cueing of the foot improves balance in the elderly female population. This is an important area of research because as stated decreased balance of the elderly is the leading cause of falls and therefore injury. Physical therapist must play an active role as health care providers in improving patient balance and therefore minimizing patient risk of falling.

\section{Hypothesis}

We hypothesize that sensory stimulation will be more effective than motor control cueing in increasing balance in elderly female patients. As well as a null hypothesis of, neither sensory stimulation nor motor control cueing will have a positive effect on balance in the female elderly population.

\section{Methods}

The study is a Quasi-Experimental Single Group Pretest-Posttest design approved by Dominican College Institutional Review Board (IRB approval \# 2018-0325-01). Participants received both interventions on two separate data collection days. The sensory stimulation intervention was performed on the first data collection day. The motor control cue was given as the second intervention on the second data collection day.

Subjects were recruited from three assisted living facilities. The sample size consisted of 40 participants between the ages of 67 to 97 years old (mean 82; SD 6.25). In order to be included in the study, subjects needed to be female and over the age of 65 years old. The exclusion criteria were as follows: resting $\mathrm{HR}<50$ bpm, resting HR $>100 \mathrm{bpm}$, oxygen saturation $<90 \%$, BP $<90 / 60 \mathrm{mmHg}, \mathrm{BP}>$ $140 / 90 \mathrm{mmHg}$, recent surgery or fracture, severe neuropathy, weight-bearing restrictions, and a Mini-Mental State Exam score (MMSE) of $\leq 20$ [7]. Written consent forms were signed from all participants prior to beginning the study. This statement was provided to participants with information regarding the procedures of the study and to inform patients that they were allowed to stop testing at any point during the study.

Research materials required for this study included a blood pressure cuff, stethoscope, pulse oximeter, a yardstick for the Functional Reach Test, the Biodex BioSway Portable Balance System, a reflex hammer to provide sensory stimulation, printed versions of the MMSE. A stopwatch was required to limit patient practice time and sensory stimulation time respectively.

On the first day of data collection, Researcher One screened participants for 
exclusion criteria by providing participants with a demographics sheet. Researcher One then distributed the MMSE to each participant and took vitals for each participant including; heart rate, blood pressure and peripheral oxygen capillary saturation rate. Vitals were taken on day 1 and day 2 prior to pre and post testing. Researcher One also provided participant identifications including the participants initials followed by a number in order to maintain confidentiality. This identification was used to save results on the Biodex Biosway Portable Balance System and log participant paperwork. Researcher Two collected baseline balance information on the Biodex Biosway Portable Balance system (Biodex Medical System, Inc., Shirley, New York) first and then Researcher One collected the Functional Reach baseline measurement. The Sensory stimulation intervention was performed, and outcome measures were collected immediate after intervention.

The second data collection day was scheduled for a week following the first data collection day. Same tests and assessments were done on the second data collection day prior motor cue intervention. Researcher Three provided each participant with a motor control cue and practiced with motor cue exercise for 3 minutes. Post-test assessments were collected after motor cue intervention.

Neither participants nor researchers were blinded to the interventions. Guarding of participants while assessments of balance were being performed was crucial to ensure patient safety and was stressed throughout the duration of participant testing.

Cognitive assessment: The Mini-Mental State Examination (MMSE) was be used to determine the participant's mental status and exclude any participants with cognitive impairments. This examination is a commonly administered screening tool for dementia and any cognitive impairment. Research shows that the MMSE is effective as an exclusion tool [8]. The MMSE contained 11 questions/activities, which were asked in the order listed on the sheet. There were 30 possible points. One point was scored for each correct answer response within each activity or question. This questionnaire examined the following functions: orientation, registration, attention, recall, and language. Administration of the test takes approximately 5 - 10 minutes [9]. The inclusion criteria for the participants to be included in the study, was to score a 21 or greater, signifying that cognition will not impact the results or outcomes of this study.

Functional reach test: The Functional Reach Test measures the distance a person can reach forward while maintaining a fixed base of support. When performing this test, the subject was asked to have their feet shoulder width apart in a comfortable position. The subject was then instructed to raise their arms parallel to the floor, make a fist, and reach forward as far as possible keeping both feet planted on the floor. With the third metacarpal head as a reference point, the distance in inches, was then measured from start point to end point. The test was being repeated 3 times and the average was taken. The test-retest and inter-rater reliability for the FRT has been shown to be excellent for adults be- 
tween the ages of 20 to 87 years old [10]. In addition, the intra-class correlation coefficient for the functional reach test is 0.99 [11].

Balance assessment: The Biodex Biosway Portable Balance System is a valid and reliable tool used for balance assessment and balance training (Biodex Medical System Inc., Shirley, New York) [12]. The intraclass correlation coefficient for the Biodex Biosway is 0.81 with a $95 \%$ confidence interval [12]. The Postural Stability Test was utilized for this research study to assess participants balance on a static surface both prior to interventions as a baseline measure and post-intervention as an outcome measure. The Postural Stability Test monitored participant's ability to maintain their Center of Balance (COB). The Overall Sway Index was utilized for data analysis and included anterior-posterior and medial-lateral sway. The participant's score revealed the amount of deviations from their COB, therefore the goal was to achieve a lower score [13]. When the Biodex Biosway was set up, the procedure was explained to the participant, the participant stood on the platform, and there was a touch start for the cursor to appear on the screen. Three test trials were completed, average value is used for statistical analysis.

\section{Interventions:}

Sensory stimulation intervention: The first intervention given to the participants on data collection day one was sensory stimulation to the sole of the foot. Researchers Priplata et al. [3] and Mildren and Bent [4] found that vibration to the sole of the foot increased proprioception and decreased postural sway. Our research utilized a different sensory stimulation technique as seen in Figure 1. Researcher Two used the metal end of a reflex hammer to stroke the sole of the foot from the heel to the metatarsal heads in 30 second intervals for a total of one minute on each foot. The participant was asked to rate the level of pressure they feel from the Reflex hammer on their foot from 1 - 10, with 1 being the participant did not feel the reflex hammer to 10 being it was painful. To ensure uniformity among patients, pressure was rated at a 7 by participants. This technique to provide sensory stimulation was used as it requires minimal equipment (vibration requires extensive equipment) and has low risk of injury (heat or cold may cause burns).

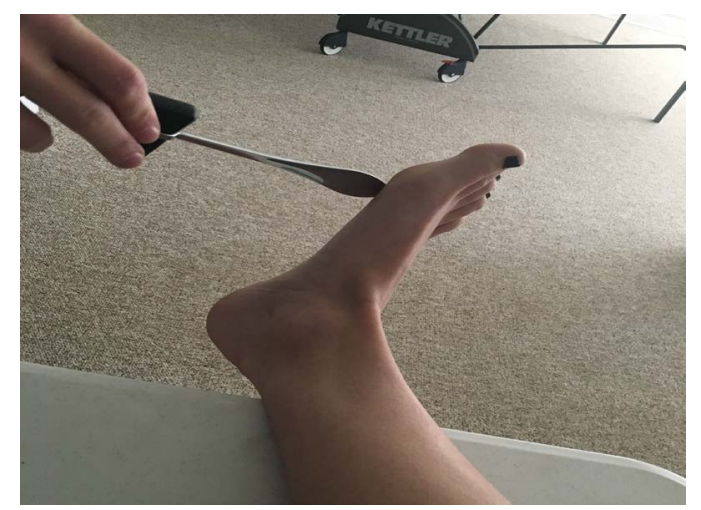

Figure 1. The sensory stimulation application using a reflex hammer. 
Motor cueing: The second intervention performed on participants on day two was the "short foot" motor control cue. Participants were instructed to think of shortening the foot by bringing the toes closer to the heel of the foot and lifting the arch. Jung et al. [6] described performing the "short foot" as "pulling the metatarsal heads toward the heel, while the long toe flexors are relaxed". This was the most effective in activating the abductor hallicus, which acts as a stabilizer during static balance. Our rationale for using the cue of "bringing the toes closer to the heel and lifting the arch" was simplified from the cue provided by Jung et al. [6]. The participants had 1 minute to practice standing using this cue before returning for the final outcome measurements using the Biodex Biosway Portable Balance System and the Functional Reach Test measurement.

The statistical analyses were completed using the IBM Statistical Package for the Social Sciences (SPSS) Version 25. The data was analyzed utilizing pre-test and post-test scores of balance obtained with the Biodex Biosway and the functional reach test. The pre-test post-test analysis was performed using the Repeated Measures Analysis of Variance (ANOVA). The confidence interval (CI) was set at $95 \%$ and the alpha level for significance was $\mathrm{p}<0.05$.

\section{Results}

Initially 40 participants were contacted to participate in this study. The data of thirty-two participants was used to complete this study. Four participants were excluded due to sensory deficits (peripheral neuropathy), three participants due to excessively high blood pressure, and one participant due to a foot injury prior to the study. Participants' demographics collected on the first day of data collection. Average age was $82.0(\mathrm{SD}=6.25)$ years, MMSE score was $28.7(\mathrm{SD}=12.6)$, and daily walking time was $24(\mathrm{SD}=8.4)$ minutes. The $75 \%$ of the participants were not using assistive device. The participants' demographic can be found on Table 1.

With sensory stimulation intervention, balance (postural sway) as the dependent variable, there was a significant difference between pretest and post-test balance scores, $t(31)=2.089, \mathrm{p}=0.045, d=0.37$ [95\% CI $0.22-1.83$ ]. The results $(M \pm \mathrm{SD})$ showed that postural sway declined from pretest $5.92 \pm 3.05$ to posttest $(5.00 \pm 2.92)$. With motor control intervention, balance (postural sway) as the dependent variable, there was a significant difference between pretest and post-test scores, $t(31)=-2.578, \mathrm{p}=0.015, d=-0.46$ [95\% CI $-2.04-0.24]$. The results $(M \pm \mathrm{SD})$ showed that postural sway increased from pretest $(5.52 \pm 3.50)$ to posttest $(6.66 \pm 4.06)$ (Figure 2).

With sensory stimulation intervention, functional reach test as the dependent variable, there was a significant difference between pretest and post-test measurements, $t(31)=-3.942, \mathrm{p}<0.001, d=-0.70$ [95\% CI $-1.05--0.33]$. The results $(M \pm \mathrm{SD})$ showed that functional reach test distance increased from pretest $(8.01 \pm 2.55)$ to posttest $(8.70 \pm 2.66)$. With motor control intervention, functional reach test as the dependent variable, there was a significant difference between pretest and post-test measurements, $t(31)=-3.135, \mathrm{p}=0.004, d=-0.55$ 
[95\% CI $-0.89--0.19]$. The results $(M \pm \mathrm{SD})$ showed that functional reach test distance increased from pretest $(8.47 \pm 2.34)$ to posttest $(9.01 \pm 2.37)$ (Figure 3$)$.

Table 1. Participants' demographics.

\begin{tabular}{cccccc}
\hline & Mean & SD & Min & Max & n (\%) \\
\hline Age (year) & 82.0 & 6.25 & 67 & 96 & \\
Weight (pound) & 154.9 & 27.59 & 107 & 220 & \\
Height (inches) & 62.7 & 2.55 & 57 & 71 & \\
Mini Mental State Examination Score & 28.7 & 1.26 & 26 & 30 & \\
Sedentary Time (minute) & 125 & 85.88 & & & \\
Walking Time (minute) & 24 & 8.40 & & & \\
Assistive Device (AD) Usage & & & & & \\
No AD & & & & & $24(75)$ \\
1 AD & & & & & $4(12.5)$ \\
2 AD & & & & & $4(12.5)$ \\
\hline
\end{tabular}

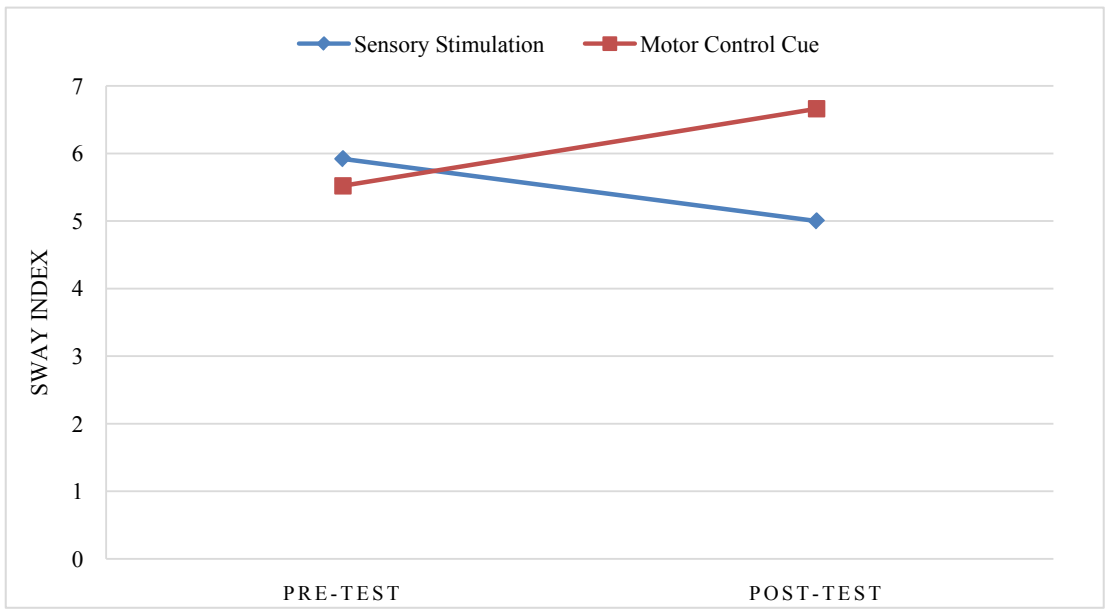

Figure 2. Pre- and post-test postural sway index of sensory stimulation and motor control.

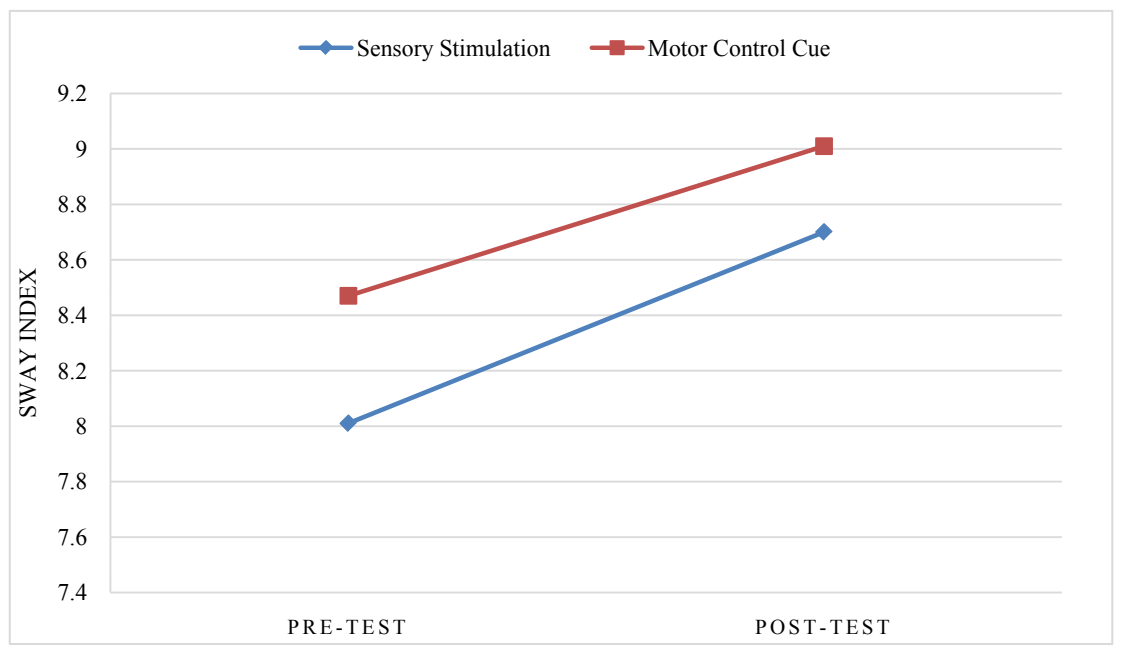

Figure 3. Pre- and post-test functional reach test scores of sensory stimulation and motor control. 


\section{Discussion}

This study aimed to investigate the immediate effect of sensory stimulation and motor control cueing on standing balance in older females. After providing both sensory stimulation and motor control cueing interventions to the 32 participants, the results showed that sensory stimulation was more effective than motor control cueing in improving balance as demonstrated by improved balance measures for both outcome measures.

Several studies have looked at age related changes affecting touch sensation and its impact on postural stability in the elderly [14]. Peripheral sensation seems to be the single most important factor in maintenance of static postural stability and reduced foot tactile sense in the elderly contributes to postural instability [15]. Our research used sensory stimulation to prime the foot before engaging in balance activities, however, current literature reveals studies which utilize vibrating insoles during balance activities to increase proprioception [3] [4]. Currently, there are no research studies that apply tactile cueing before engaging in activities requiring proprioception and balance in weight bearing. Thus, we hypothesized that sensory stimulation of the plantar surface of the foot will improve postural stability in elderly females. We found that older females showed decreased postural sway and increased functional reach test distance on the Biodex balance machine and FRT after applying 1-minute sensory stimulation on the sole of the foot. These results suggest that application of tactile plantar stimulation may compensate a loss of superficial plantar sensitivity and immediately improve both static and dynamic balance after application.

Mulligan and Cook [16] stated that using short foot exercises improved dynamic postural control. Lynn et al. [17] concluded that strengthening the foot intrinsic muscles through short foot exercises improved dynamic postural control by increasing activation of the foot intrinsic muscles during dynamic postural control, but not static postural control. When utilizing the "short foot" cue, the goal is to heighten the medial longitudinal arch and activate the intrinsic foot musculature in order to provide stability throughout the lower kinetic chain and therefore improve balance and postural control [18]. Our research findings support the studies conducted by Mulligan and Cook [16] and Lynn et al. [17] demonstrating that utilizing the short foot motor control cue improves dynamic postural control as noted by the Functional Reach Test, and not static postural control on the Biodex. Participants had difficulty understanding the concept and while a majority demonstrated understanding following the minute of practice, some did not. Therefore, we conclude that the complexity of the motor control cue would be one of the reasons why the participants' static balance didn't improve.

Findings of this research are important for health care providers because of the prevalence of the geriatric population requiring physical therapy services. Age-related balance losses are a common complaint in those over 65 years old, putting this population at a significantly higher risk of falling. Thus, the impor- 
tance in maintaining adequate balance is needed to decrease risk of falls and associated consequences. This study is providing therapists with the knowledge of another potential intervention to address balance deficits. Physical Therapist can utilize sensory stimulation for patients with decreased postural control resulting in poor balance. By improving balance and postural control in the elderly female population a decrease in fall risk and the secondary impairments associated with falls can be prevented.

Having found a statistically significant result in the sensory stimulation group for balance is an added strength to our research study. In addition, there was one week between interventions and data collection days to prevent a maturation threat or interaction between treatment session threat. Further research needs to be performed with a larger randomized population with different utilization of instruments to determine the generalizability of these findings, as well as the long-term effectiveness of sensory stimulation on improving balance.

\section{Conclusion}

Based upon the results, a conclusion can be made that sensory stimulation to the sole of the foot is more effective than motor control cueing of the "short foot", in improving both static and dynamic balance in the elderly female population. Sensory stimulation has shown to be a feasible, simple, and a cost-efficient intervention to address balance in this specific population. In the clinic, physical therapists can utilize sensory stimulation for patients with decreased postural control resulting in poor balance. By improving balance and postural control in the elderly female population a decrease in fall risk and the secondary impairments associated with falls can be prevented.

\section{Statement}

Institutional Review Board Approval (\# 2018-0325-01).

\section{Conflicts of Interest}

The authors declare no conflicts of interest regarding the publication of this paper.

\section{References}

[1] Iwasaki, S. and Yamasoba, T. (2015) Dizziness and Imbalance in the Elderly: Age-Related Decline in the Vestibular System. Aging and Disease, 6, 38-47. https://doi.org/10.14336/AD.2014.0128

[2] Lee, A., Lee, K.W. and Khang, P. (2013) Preventing Falls in the Geriatric Population. The Permanente Journal, 17, 37-39. https://doi.org/10.7812/TPP/12-119

[3] Priplata, A.A., Niemi, J.B., Harry, J.D., Lipsitz, L.A. and Collins, J.J. (2003) Vibrating Insoles and Balance Control in Elderly People. The Lancet, 362, 1123-1124. https://doi.org/10.1016/S0140-6736(03)14470-4

[4] Mildren, R.L. and Bent, L.R. (2016) Vibrotactile Stimulation of Fast-Adapting Cutaneous Afferents from the Foot Modulates Proprioception at the Ankle Joint. Journal of Applied Physiology, 120, 855-864. 
https://doi.org/10.1152/japplphysiol.00810.2015

[5] Rothermel, S.A., Hale, S.A., Hertel, J. and Denegar, C.R. (2004) Effect of Active Foot Positioning on the Outcome of a Balance Training Program. Physical Therapy in Sport, 5, 98-103. https://doi.org/10.1016/j.ptsp.2004.02.002

[6] Jung, D., Kim, M., Koh, E., Kwon, O., Cynn, H. and Lee, W. (2011) A Comparison in the Muscle Activity of the Abductor Hallucis and the Medial Longitudinal Arch Angle during Toe Curl and Short Foot Exercises. Physical Therapy in Sport, 12, 30-35. https://doi.org/10.1016/j.ptsp.2010.08.001

[7] Folstein, M.F., Folstein, S.E. and McHugh, P.R. (1975) "Mini-Mental State": A Practical Method for Grading the Cognitive State of Patients for the Clinician. Journal of Psychiatric Research, 12, 189-198. https://doi.org/10.1016/0022-3956(75)90026-6

[8] Xu, X., Chong, E., Hilal, S., Ikram, M.K., Venketasubramanian, N. and Chen, C. (2015) Beyond Screening: Can the Mini-Mental State Examination Be Used as an Exclusion Tool in a Memory Clinic? Diagnostics, 5, 475-486.

https://doi.org/10.3390/diagnostics5040475

[9] Kurlowicz, L. and Wallace, M. (1999) The Mini-Mental State Examination (MMSE). Journal of Gerontological Nursing, 25, 8-9. https://doi.org/10.3928/0098-9134-19990501-08

[10] Duncan, P.W., Weiner, D.K., Chandler, J. and Studenski, S. (1990) Functional Reach: A New Clinical Measure of Balance. Journal of Gerontology, 45, M192-M197. https://doi.org/10.1093/geronj/45.6.M192

[11] Bennie, S., Bruner, K., Dizon, A., Fritz, H., Goodman, B. and Peterson, S. (2003) Measurements of Balance: Comparison of the Timed "Up and Go" Test and Functional Reach Test with the Berg Balance Scale. Journal of Physical Therapy Science, 15, 93-97. https://doi.org/10.1589/jpts.15.93

[12] Biodex Medical Systems, Inc. Biosway Portable Balance System: Operation Manual. Author, New York. http://www.biodex.com/sites/default/files/950460man_10202revd.pdf

[13] Schmitz, R. and Arnold, B. (1998) Intertester and Intratester Reliability of a Dynamic Balance Protocol Using the Biodex Stability System. Journal of Sport Rehabilitation, 7, 95-101. https://doi.org/10.1123/jsr.7.2.95

[14] Lord, S.R., Clark, R.D. and Webster, I.W. (1991) Postural Stability and Associated Physiological Factors in a Population of Aged Persons. Journal of Gerontology, 46, M69-M76. https://doi.org/10.1093/geronj/46.3.M69

[15] Tanaka, T., Noriyasu, S., Ino, S., et al. (1996) Objective Method to Determine the Contribution of the Great Toe to Standing Balance and Preliminary Observations of Age-Related Effects. IEEE Transactions on Rehabilitation Engineering, 4, 84-90. https://doi.org/10.1109/86.506405

[16] Mulligan, E.P. and Cook, P.G. (2013) Effect of Plantar Intrinsic Muscle Training on Medial Longitudinal Arch Morphology and Dynamic Function. Manual Therapy, 18, 425-430. https://doi.org/10.1016/j.math.2013.02.007

[17] Lynn, S.K., Padilla, R.A. and Tsang, K.K. (2012) Differences in Static- and Dynamic-Balance Task Performance after 4 Weeks of Intrinsic-Foot-Muscle Training: The Short-Foot Exercise versus the Towel-Curl Exercise. Journal of Sports Rehabilitation, 21, 327-333. https://doi.org/10.1123/jsr.21.4.327

[18] Mignogna, A.C., Welsch, A.L. and Hoch, C.M. (2016) The Effect of Short Foot Exercises on Postural Control: A Critically Appraised Topic. International Journal of Athletic Therapy and Training, 21, 8-12. https://doi.org/10.1123/ijatt.2016-0049 